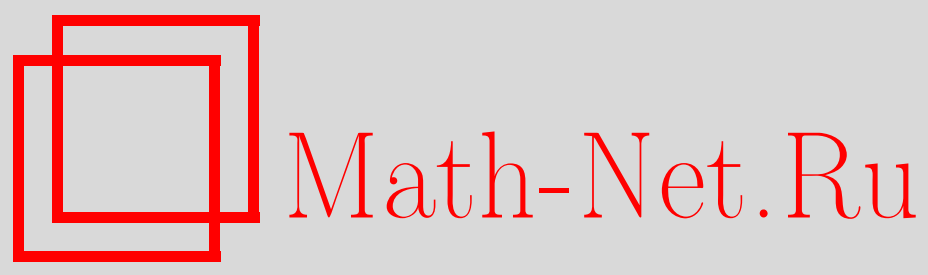

Г. А. Свиридюк, Т. Г. Сукачева, О разрешимости нестационарной задачи динамики несжимаемой вязкоупругой жидкости, Матем. заметки, 1998, том 63, выпуск 3, 442-450

DOI: https://doi.org/10.4213/mzm1301

Использование Общероссийского математического портала Math-Net.Ru подразумевает, что вы прочитали и согласны с пользовательским соглашением http://www . mathnet.ru/rus/agreement

Параметры загрузки:

IP : 3.81 .55 .215

26 апреля 2023 г., $17: 14: 33$

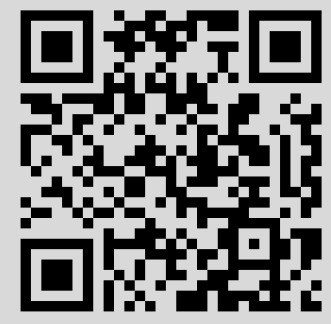




\section{О РАЗРЕШИМОСТИ НЕСТАЦИОНАРНОЙ ЗАДАЧИ ДИНАМИКИ НЕСЖИМАЕМОЙ ВЯЗКОУПРУГОЙ ЖИДКОСТИ}

\section{Г.А. Свиридюк, Т.Г. Сукачёва}

Исследована локальная разрешимость задачи Коши-Дирихле для системы уравнений

$$
\begin{gathered}
\left(1-\varkappa \nabla^{2}\right) \mathbf{v}_{t}=\nu \nabla^{2} \mathbf{v}-(\mathbf{v} \cdot \nabla) \mathbf{v}-\nabla p+\mathbf{f}(t), \\
0=-\nabla(\nabla \cdot \mathbf{v})
\end{gathered}
$$

моделирующей динамику несжимаемой вязкоупругой жидкости типа Кельвина-Фойгта. Получено описание конфигурационного пространства этой задачи.

Библиографоия: 14 названий.

Система уравнений Осколкова [1]

$$
\begin{gathered}
\left(1-\varkappa \nabla^{2}\right) \mathbf{v}_{t}=\nu \nabla^{2} \mathbf{v}-(\mathbf{v} \cdot \nabla) \mathbf{v}-\nabla p+\mathbf{f}(t), \\
0=\nabla \cdot \mathbf{v}
\end{gathered}
$$

моделирует динамику несжимаемой вязкоупругой жидкости Кельвина-Фойгта. Пусть $\Omega \subset \mathbb{R}^{n}, n=2,3,4,-$ ограниченная область с границей $\partial \Omega$ класса $C^{\infty}$. До сих пор нами [2], [3] рассматривалась задачи Коши-Дирихле

$$
\begin{gathered}
\mathbf{v}(x, 0)=\mathbf{v}_{0}(x), \quad x \in \Omega, \\
\mathbf{v}(x, t)=0, \quad(x, t) \in \partial \Omega \times \mathbb{R},
\end{gathered}
$$

для системы (1) в предположении, что свободньй член $\mathbf{f}=\left(f_{1}, \ldots, f_{n}\right)$ не зависит от времени, т.е. $f_{k}=f_{k}(x), x \in \Omega, k=1, \ldots, n$. Такое предположение, во-первых, снижает эвристическую ценность модели, а во-вторых, уступает по обшности [4], где задача $(1),(2)$ рассмотрена в случае $f_{k}=f_{k}(x, t)$ и $\varkappa^{-1}>-\lambda_{1}\left(\lambda_{1}-\right.$ наименьшее собственное значение задачи Дирихле для оператора Лапласа в области $\Omega$ ). С другой стороны, подход, предложенньй в [5], позволяет рассмотреть задачу $(1),(2)$ в полной общности, т.е. при нестационарном свободном члене $\mathbf{f}=\mathbf{f}(x, t)$ и любых значениях параметра $\varkappa \in \mathbb{R}$, характеризуюшего упругие свойства жидкости. (Заметим, что отрицательные значения

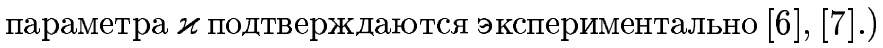

Чтобы избежать вопроса о том, как несжимаемая жидкость может быть упругой, систему (1) рассматривают как предельньй (при $\varepsilon \downarrow 0)$ случай системы

$$
\begin{gathered}
\left(1-\varkappa \nabla^{2}\right) \mathbf{v}_{t}=\nu \nabla^{2} \mathbf{v}-(\mathbf{v} \cdot \nabla) \mathbf{v}-\nabla p+\mathbf{f}, \\
\varepsilon p_{t}=-\nabla \cdot \mathbf{v},
\end{gathered}
$$


моделирующей динамику слабосжимаемой вязкоупругой жидкости типа КельвинаФойгта. Производя в (3) замену $\mathbf{p}=\nabla p$ и полагая $\varepsilon=0$, придем к системе

$$
\begin{gathered}
\left(1-\varkappa \nabla^{2}\right) \mathbf{v}_{t}=\nu \nabla^{2} \mathbf{v}-(\mathbf{v} \cdot \nabla) \mathbf{v}-\mathbf{p}+\mathbf{f} \\
0=-\nabla(\nabla \cdot \mathbf{v}) .
\end{gathered}
$$

Несмотря на то, что оператор $\nabla$ имеет ядро, натянутое на константу, мы не получим в задаче (4), (2) новых решений по сравнению с задачей (1), (2), благодаря краевому условию (2). Замена $\mathbf{p}=\nabla p$ объясняется тем, что в большинстве гидродинамических задач [8] рассмотрение градиента давления предпочтительнее рассмотрения давления.

Нас будет интересовать локальная однозначная разрешимость задачи (2), (4). Эту задачу удобно рассматривать в рамках теории уравнений типа Соболева. Поэтому сначала мы развиваем некий формализм, изложенньй в первой части статьи. Основной результат здесь - существование квазистационарных траекторий [5] полулинейного нестационарного уравнения типа Соболева. Затем задачу $(2),(4)$ рассматриваем в рамках этого формализма.

1. Формальная схема. Пусть $\mathfrak{U}$ и $\mathfrak{F}$ - банаховы пространства; заданы операторы $L \in \mathscr{L}(\mathfrak{U} ; \mathfrak{F})$ и $M \in C^{\infty}(\mathfrak{U} ; \mathfrak{F})$, функция $f: \mathbb{R} \rightarrow \mathfrak{F}$. Поставим задачу Коши

$$
u(0)=u_{0}
$$

для полулинейного нестационарного уравнения типа Соболева

$$
L \dot{u}=M(u)+f .
$$

Линейньй оператор $L: \mathfrak{U} \rightarrow \mathfrak{F}$ назьвается бирасщепляющим [9], если его ядро $\operatorname{ker} L$ и образ $\operatorname{im} L$ дополняемы в пространствах $\mathfrak{U}$ и $\mathfrak{F}$ соответственно. Пусть оператор $L$ бирасщепляющий; обозначим через $M_{u_{0}}^{\prime} \in \mathscr{L}(\mathfrak{U} ; \mathfrak{F})$ производную Фреше оператора $M$ в точке $u_{0} \in \mathfrak{U}$ и введем в рассмотрение цепочки $M_{u_{0}}^{\prime}$-nрисоединенных векторов операmора $L[10]$, которые будем выбирать из некоторого дополнения $\operatorname{coim} L=\mathfrak{U} \ominus \operatorname{ker} L \mathrm{k}$ ядру $\operatorname{ker} L$. Введем в рассмотрение условие:

A1) независимо от выбора coim $L$ цепочка $M_{u_{0}}^{\prime}$-присоединенных векторов любого вектора $\varphi \in \operatorname{ker} L \backslash\{0\}$ содержит точно $p$ элементов.

Обозначим через $\widetilde{L}$ сужение оператора $L$ на $\operatorname{coim} L$. В силу теоремы Банаха о замкнутом графике оператор $\widetilde{L}: \operatorname{coim} L \rightarrow \operatorname{im} L-$ топлинейный изоморфизм. Положим $\mathfrak{U}_{0}^{0}=\operatorname{ker} L$ и построим множества $\mathfrak{U}_{q}^{0}=\widetilde{A}^{q}\left[\mathfrak{U}_{0}^{0}\right], q=1, \ldots, p$, где $\widetilde{A}=\widetilde{L}^{-1} M_{u_{0}}^{\prime}$. Очевидно, множества $\mathfrak{U}_{q}^{0} \subset \operatorname{coim} L$ являются линейными пространствами. Следовательно, образ $\mathfrak{F}_{p}^{0}=M_{u_{0}}^{\prime}\left[\mathfrak{U}_{p}^{0}\right]$ также является линейным пространством, причем $\mathfrak{F}_{p}^{0} \cap \operatorname{im} L=\{0\}$ (если вьполнено условие A1)). Введем в рассмотрение еще одно условие:

A2) $\mathfrak{F}_{p}^{0} \oplus \operatorname{im} L=\mathfrak{F}$.

Обозначим через $Q_{p}: \mathfrak{F} \rightarrow \mathfrak{F}_{p}^{0}$ проектор вдоль $\operatorname{im} L$ и построим оператор $A=\widetilde{L}^{-1} \times$ $\left(I-Q_{p}\right) M_{u_{0}}^{\prime}$. Заметим, что $A\left[\mathfrak{U}_{q}^{0}\right]=\mathfrak{U}_{q+1}^{0}, q=0,1, \ldots, p-1, A\left[\mathfrak{U}_{p}^{0}\right]=\{0\}$. Отсюда следует, что

$$
A^{q}\left[\mathfrak{U}_{r}^{0}\right]= \begin{cases}\{0\}, & q+r>p, \\ \mathfrak{U}_{q+r}^{0}, & q+r \leqslant p .\end{cases}
$$


Построим оператор $D$, равньй сужению оператора $Q_{p} M_{u_{0}}^{\prime} A^{p}: \mathfrak{U} \rightarrow \mathfrak{F}_{p}^{0}$ на $\mathfrak{U}_{0}^{0}$. По построению $D\left[\mathfrak{U}_{0}^{0}\right]=\mathfrak{F}_{p}^{0}$ и $D \in \mathscr{L}\left(\mathfrak{U}_{0}^{0} ; \mathfrak{F}_{p}^{0}\right)$. Кроме того, $\operatorname{ker} D=\{0\}$, ибо в противном случае вектор $\varphi \in \operatorname{ker} D \backslash\{0\} \subset \operatorname{ker} L \backslash\{0\}$ имел бы бесконечную цепочку $\left\{\varphi_{1}, \varphi_{2}, \ldots, \varphi_{p}\right.$, $0, \ldots\} M_{u_{0}}^{\prime}$-присоединенных векторов. В силу упоминавшейся теоремы Банаха оператор $D: \mathfrak{U}_{0}^{0} \rightarrow \mathfrak{F}_{p}^{0}$ - топлинейньй изоморфизм.

Обозначим через $P_{0}: \mathfrak{U} \rightarrow \mathfrak{U}_{0}^{0}$ проектор вдоль $\operatorname{coim} L$ и построим операторы $P_{q}=$ $A^{q} D^{-1} Q_{p} M_{u_{0}}^{\prime} A^{p-q}, q=1, \ldots, p$. Операторы $P_{q}: \mathfrak{U} \rightarrow \mathfrak{U}_{q}^{0}$-проекторы. Действительно, $\operatorname{im} P_{q}=\mathfrak{U}_{q}^{0}, P_{q} \in \mathscr{L}(\mathfrak{U})$ и

$$
P_{q}^{2}=A^{q}\left(D^{-1}\left(Q_{p} M_{u_{0}}^{\prime} A^{p}\right)\right) D^{-1} Q_{p} M_{u_{0}}^{\prime} A^{p-q}=P_{q}
$$

по определению оператора $D$. Кроме того, в силу (7) и определения проектора $P_{0}$

$$
P_{q} P_{r}=P_{r} P_{q}=0, \quad q, r=0,1, \ldots, p, \quad q \neq r .
$$

Положим

$$
\mathfrak{U}^{0}=\bigoplus_{q=0}^{p} \mathfrak{U}_{q}^{0}, \quad P=\sum_{q=0}^{p} P_{q} .
$$

Оператор $P \in \mathscr{L}(\mathfrak{U})$ - проектор, im $P_{0}=\mathfrak{U}^{0}$. Положим $\mathfrak{U}^{1}=\operatorname{ker} P$. Тогда $\mathfrak{U}=\mathfrak{U}^{0} \oplus \mathfrak{U}^{1}$.

Введем в рассмотрение линеалы $\mathfrak{F}_{q}^{0}=M_{u_{0}}^{\prime}\left[\mathfrak{U}_{q}^{0}\right], q=0,1, \ldots, p-1$, и построим оператор $B=M_{u_{0}}^{\prime} \widetilde{L}^{-1}\left(I-Q_{p}\right)$. Поскольку $B\left[\mathfrak{F}_{q}^{0}\right]=\mathfrak{F}_{q+1}^{0}, q=0,1, \ldots, p-1, B\left[\mathfrak{F}_{p}^{0}\right]=\{0\}$, выполнено

$$
B^{q}\left[\mathfrak{F}_{r}^{0}\right]= \begin{cases}\{0\}, & q+r>p, \\ \mathfrak{F}_{q+r}^{0}, & q+r \leqslant p .\end{cases}
$$

Из (8) аналогично сказанному вьше следует, что операторы $Q_{q}=B^{q} M_{u_{0}}^{\prime} D^{-1} Q_{p} B^{p-q}$, $q=0,1, \ldots, p-1$, являются проекторами на $\mathfrak{F}_{q}^{0}$, причем

$$
Q_{q} Q_{r}=Q_{r} Q_{q}=\mathbf{0}, \quad q, r=0,1, \ldots, p, \quad q \neq r .
$$

Положим

$$
\mathfrak{F}^{0}=\bigoplus_{q=0}^{p} \mathfrak{F}_{q}^{0}, \quad Q=\sum_{q=0}^{p} Q_{q} .
$$

Оператор $Q \in \mathscr{L}(\mathfrak{F})$ - проектор, поэтому $\mathfrak{F}=\mathfrak{F}^{0} \oplus \mathfrak{F}^{1}$, где $\mathfrak{F}^{0}=\operatorname{im} Q, \mathfrak{F}^{1}=\operatorname{ker} Q$.

Заметим, что по построению

$$
L A^{q} D^{-1} Q_{p}=B^{q-1} M_{u_{0}}^{\prime} D^{-1} Q_{p}, \quad q=1, \ldots, p .
$$

Кроме того,

$$
B L=M_{u_{0}}^{\prime}\left(I-P_{0}\right)
$$

Из $(9),(10)$ при $q=1, \ldots, p$ получаем

$$
\begin{aligned}
L P_{q} & =L P_{q}\left(I-P_{0}\right)=L A^{q} D^{-1} Q_{p} M_{u_{0}}^{\prime} A^{p-q}\left(I-P_{0}\right) \\
& =B^{q-1} M_{u_{0}}^{\prime} D^{-1} Q_{p} B^{p-q} M_{u_{0}}^{\prime}\left(I-P_{0}\right)=Q_{q-1} L .
\end{aligned}
$$

Перепишем уравнение (7) в виде

$$
L \dot{u}=M_{u_{0}}^{\prime} u+F(u)+f,
$$


где оператор $F=M-M_{u_{0}}^{\prime} \in C^{\infty}(\mathfrak{U} ; \mathfrak{F})$ по построению. Действуя на уравнение $(12)$ последовательно проекторами $Q_{q}, q=0,1, \ldots, p$, и $I-Q$, получим в силу (11) эквивалентную систему:

$$
\begin{aligned}
L \dot{u}_{1}^{0} & =M_{u_{0}}^{\prime} u_{0}^{0}+F_{0}(u)+f_{0}^{0} \\
\ldots \ldots \ldots \ldots \ldots \ldots \ldots \ldots & \ldots \ldots \ldots \\
L \dot{u}_{p}^{0} & =M_{u_{0}}^{\prime} u_{p-1}^{0}+F_{p-1}(u)+f_{p-1}^{0} \\
0 & =M_{u_{0}}^{\prime} u_{p}^{0}+F_{p}(u)+f_{p}^{0} \\
L \dot{u}^{1} & =(I-Q) M(u)+f^{1}
\end{aligned}
$$

где $u_{q}^{0} \in \mathfrak{U}_{q}^{0}, f_{q}^{0} \in \mathfrak{F}_{q}^{0}, F_{q}(u)=Q_{q} F(u)+Q_{q} M_{u_{0}}^{\prime} u^{1}, q=0,1, \ldots, p, u^{1} \in \mathfrak{U}^{1}, f^{1} \in \mathfrak{F}^{1}$. Итак, доказана

Лемма 1. Пусть операторы $L \in \mathscr{L}(\mathfrak{U} ; \mathfrak{F}), M \in C^{\infty}(\mathfrak{U} ; \mathfrak{F})$, причем $L-$ бирасщепляющий оператор, и выполнены условия А1) и А2). Тогда уравнение (6) эквивалентно системе (13).

ЗАмЕчАнИЕ 1 . В условиях леммы 1 оператор $M L$-ограничен в точке $u_{0}[5]$, причем $\infty$ - полюс порядка $p$ оператор-функции $\left(\mu L-M_{u_{0}}^{\prime}\right)^{-1}$.

Займемся теперь поисками решения задачи (6), (7). Решением этой задачи называется вектор-функция $u \in C^{\infty}\left(\left(-t_{0}, t_{0}\right) ; \mathfrak{U}\right), t_{0}=t_{0}\left(u_{0}\right)>0$, удовлетворяющая уравнению (6) и условию (5). На этом пути нас поджидают две трудности. Во-первых, как хорошо известно [2], [3], [11], решения задачи $(5),(6)$ существуют не для всех $u_{0} \in \mathfrak{U}$. Во-вторых, даже в случае сушествования решения задачи (5), (6) оно может быть неединственным [5]. Чтобы преодолеть первую трудность (и частично вторую), введем следующее понятие.

ОПРЕДЕЛЕНИЕ 1. Множество $\mathfrak{B}^{t} \subset \mathfrak{U} \times \mathbb{R}$ назовем конфигурационным пространством уравнения (6), если для любой точки $u_{0} \in \mathfrak{U}$ такой, что $\left(u_{0}, 0\right) \in \mathfrak{B}^{0}$, существует единственное решение задачи $(5),(6)$, причем $(u(t), t) \in \mathfrak{B}^{t}$.

ЗАмЕчАнИЕ 2. Если $\mathfrak{B}^{t}=\mathfrak{B} \times \mathbb{R}$, где $\mathfrak{B} \subset \mathfrak{U}$, то множество $\mathfrak{B}$ называется фазовым пространством уравнения (6) (см. [2], [3], [5]).

Для того чтобы полностью устранить вторую трудность, мы ограничимся поиском квазистационарных траекторий уравнения (6), т.е. таких решений $u=u(t)$ задачи (5), $(6)$, для которых $L \dot{u}^{0} \equiv 0$ при любом $t \in\left(-t_{0}, t_{0}\right)$, где $u^{0}=P u$ [5]. Для выделения квазистационарных траекторий из множества возможных решений задачи (5), (6) потребуем выполнения ряда условий.

А3) $f_{q}^{0}(t) \equiv 0$ при любом $t \in \mathbb{R}, q=1, \ldots, p$.

Рассмотрим множество $\widetilde{\mathfrak{U}}=\left\{u \in \mathfrak{U}: u_{q}^{0}=\right.$ const, $\left.q=1, \ldots, p\right\}$. Как нетрудно видеть, $\widetilde{\mathfrak{U}}$ - полное аффинное многообразие, моделируемое подпространством $\mathfrak{U}_{0}^{0} \oplus \mathfrak{U}^{1}$. Пусть точка $u_{0} \in \widetilde{\mathfrak{U}}$; обозначим через $\mathfrak{O}_{u_{0}}$ некоторую окрестность $\mathfrak{O}_{u_{0}} \subset \widetilde{\mathfrak{U}}$ точки $u_{0}$.

А4) $F_{q}(u) \equiv 0$ при любом $u \in \mathfrak{O}_{u_{0}}, q=1, \ldots, p$.

TeOpema 1. Пусть

1) выполнены условия леммы 1 ;

2) точка $\left(u_{0}, 0\right) \in \mathfrak{B}^{0}$, дде $\mathfrak{B}^{t}=\left\{(u, t) \in \widetilde{\mathfrak{U}} \times \mathbb{R}: Q_{0}(M(u)+f(t))=0\right\}$;

3) вектор-функиия $f \in C^{\infty}(\mathbb{R} ; \mathfrak{F})$;

4) выполнены условия А3), А4). 
Тогда существует единственное решение задачи (5), (6), являющееся квазистаиионарной траекторией, причем $u(t) \in \mathfrak{B}^{t}$ при любом $t \in\left(-t_{0}, t_{0}\right)$.

ДокАЗАТЕЛьство. Предположим, что решение задачи (5), (6) найдено. Тогда в силу условий А 3 ), А4) из (13) следует, что $L \dot{u}^{0} \equiv 0$, т.е. решение является квазистационарной траекторией. Установим существование и единственность решения.

В силу леммы 1 и условий А3), А4) система (13) в окрестности $\mathfrak{O}_{u_{0}}$ редуцируется к виду

$$
\begin{aligned}
& 0=M_{u_{0}}^{\prime} u_{0}^{0}+F_{0}(u)+f_{0}^{0}, \\
& L \dot{u}^{1}=(I-Q) M(u)+f^{1} .
\end{aligned}
$$

Заметим, что по построению оператор $M_{u_{0}}^{\prime}: \mathfrak{U}_{0}^{0} \rightarrow \mathfrak{F}_{0}^{0}$ невырожден, и

$$
\left.F_{0 u}^{\prime}\right|_{u=u_{0}} \equiv \mathbf{0}
$$

где через $F_{0 u}^{\prime}$ обозначена производная $\Phi$ реше оператора $F_{0}$ в точке $u$. Отсюда в силу теоремы о неявной функции сушествует окрестность $\mathfrak{O}_{u_{0}}^{1} \subset(I-P)\left[\mathfrak{O}_{u_{0}}\right]$ и вектор-функция $\delta \in C^{\infty}\left(\mathfrak{O}_{u_{0}}^{1} \times \mathbb{R} ; \mathfrak{O}_{u_{0}}^{0}\right)$, где $\mathfrak{O}_{u_{0}}^{0}=P\left[\mathfrak{O}_{u_{0}}\right]$, такие, что

$$
u(t)=u_{0}^{0}(t)+\sum_{q=1}^{p} u_{q}^{0}+u^{1} \in \mathfrak{B}^{t} \quad \forall t \in \mathbb{R} .
$$

Здесь $u_{0}^{0}(t)=\delta\left(u^{1}, t\right)$ при любом $u^{1} \in \mathfrak{O}_{u_{0}}^{1}$, а $u_{q}^{0}=P_{q} u_{0}=$ const при $q=1, \ldots, p$.

Далее, из (11) следует, что $Q L=L P$. Это значит, что оператор $L: \mathfrak{U}^{1} \rightarrow \mathfrak{F}^{1}$. Обозначим через $L_{1}$ сужение оператора $L$ на $\mathfrak{U}^{1}$. Оператор $L_{1} \in \mathscr{L}\left(\mathfrak{U}^{1} ; \mathfrak{F}^{1}\right)$ инъективен по построению. Установим его сюръективность. Пусть $f^{1} \in \mathfrak{F}^{1}$. Тогда существует $\widetilde{u}=\widetilde{L}^{-1} f^{1} \in \operatorname{coim} L$. Предположим, что $P \widetilde{u} \neq 0$, т.е.

$$
P \widetilde{u}=\sum_{q=1}^{p} P_{q} \widetilde{u}=\sum_{q=1}^{p} \widetilde{u}_{q}^{0} \neq 0 .
$$

Тогда

$$
L \widetilde{u}=L P \widetilde{u}+L(I-P) \widetilde{u}=\sum_{q=1}^{p} L \widetilde{u}_{q}^{0}+L_{1}(I-P) \widetilde{u}=f^{1} \notin \mathfrak{F}^{1} .
$$

Противоречие. Итак, оператор $L_{1}: \mathfrak{U}^{1} \rightarrow \mathfrak{F}^{1}$ непрерьвно биективен, причем $L^{-1}$ есть сужение оператора $\widetilde{L}^{-1}$ на $\mathfrak{F}^{1}$.

Из всего сказанного следует, что система (14) на $\mathfrak{O}_{u_{0}}^{1}$ может быть редуцирована к виду

$$
\dot{u}^{1}=L^{-1}(I-Q) M\left(\delta\left(u^{1}, t\right)+\left(P-P_{0}\right) u_{0}+u^{1}\right)+g(t) \equiv \Phi\left(u^{1}, t\right),
$$

где $\Phi \in C^{\infty}\left(\mathfrak{O}_{u_{0}}^{1} \times \mathbb{R} ; \mathfrak{U}^{1}\right)$, а $g(t)=L^{-1} f^{1}(t)$. Однозначная локальная разрешимость задачи Коши $u^{1}(0)=(I-P) u_{0}$ для уравнения $(15)-$ классический результат [12]. Искомая квазистационарная траектория имеет вид $u(t)=\delta\left(u^{1}(t), t\right)+u^{1}(t)$, где $u^{1} \in$ $\left.C^{\infty}\left(-t_{0}, t_{0}\right) ; \mathfrak{O}_{u_{0}}^{1}\right)$ - решение задачи Коши для уравнения $(15)$.

ЗАмЕчаниЕ 3. Анализ доказательства теоремы 1 показывает, что в качестве начального значения можно брать не только точку $u_{0}$, но и любую из некоторой ее окрестности в $\mathfrak{B}^{0}$. Это означает, что множество $\mathfrak{B}^{t}$ является (локально) конфигурационным пространством. 
2. Интерпретация формальной схемы. Следуя [5], редуцируем задачу (2), (4) к задаче (5), (6). Для этого положим

$$
\mathfrak{U}=\mathbf{H}_{\sigma}^{2} \times \mathbf{H}_{\pi}^{2} \times \mathbf{H}_{p}, \quad \mathfrak{F}=\mathbf{H}_{\sigma} \times \mathbf{H}_{\pi} \times \mathbf{H}_{p},
$$

где $\mathbf{H}_{\sigma}$ - замыкание в норме $L^{2}(\Omega)=\left(L^{2}(\Omega)\right)^{n}$ линеала соленоидальных векторов $\left\{\mathbf{v} \in\left(C_{0}^{\infty}(\Omega)\right)^{n}: \nabla \cdot \mathbf{v}=0\right\}, \mathbf{H}_{\pi}=\mathbf{H}_{\sigma}^{1}, \mathbf{H}_{p}=\mathbf{H}_{\pi}$. Обозначим через $\Sigma: L^{2}(\Omega) \rightarrow \mathbf{H}_{\sigma}$ ортопроектор. Тогда $\Sigma \in \mathscr{L}\left(\left(W_{2}^{2}(\Omega) \cap W_{2}^{1}(\Omega)\right)^{n}\right)$. Положим im $\Sigma=\mathbf{H}_{\sigma}^{2}, \operatorname{ker} \Sigma=\mathbf{H}_{\pi}^{2}$. Операторы $L, M: \mathfrak{U} \rightarrow \mathfrak{F}$ определим формулами

$$
L:=\left(\begin{array}{ccc}
\Sigma A_{\varkappa} \Sigma & \Sigma A_{\varkappa} \Pi & \mathbf{0} \\
\Pi A_{\varkappa} \Sigma & \Pi A_{\varkappa} \Pi & \mathbf{0} \\
\mathbf{0} & \mathbf{0} & \mathbf{0}
\end{array}\right)
$$

где $\Pi=I-\Sigma, A_{\varkappa}=1-\varkappa \nabla^{2}$;

$$
M(u):=\left(\begin{array}{c}
\Sigma B\left(u_{\sigma}+u_{\pi}\right) \\
\Pi B\left(u_{\sigma}+u_{\pi}\right)-u_{p} \\
C\left(u_{\sigma}+u_{\pi}\right)
\end{array}\right)
$$

где $B\left(u_{\sigma}+u_{\pi}\right):=\nu \nabla^{2}\left(u_{\sigma}+u_{\pi}\right)-\left(\left(u_{\sigma}+u_{\pi}\right) \cdot \nabla\right)\left(u_{\sigma}+u_{\pi}\right), C\left(u_{\sigma}+u_{\pi}\right):=-\nabla\left(\nabla \cdot\left(u_{\sigma}+u_{\pi}\right)\right)$, $u=\left(u_{\sigma}, u_{\pi}, u_{p}\right)$.

Лемма 2. Пусть пространства $\mathfrak{U}, \mathfrak{F}$ определены формулами (16), причем $n=$ $2,3,4$, a операторы $L, M: \mathfrak{U} \rightarrow \mathfrak{F}$ формулами (17), (18). Тогда

1) оператор $L \in \mathscr{L}(\mathfrak{U} ; \mathfrak{F})$, причем если $\varkappa^{-1} \notin \sigma\left(-\nabla^{2}\right)$, mo $\operatorname{ker} L=\{0\} \times\{0\} \times \mathbf{H}_{p}$, $\operatorname{im} L=\mathbf{H}_{\sigma} \times \mathbf{H}_{\pi} \times\{0\}$

2) onepamop $M \in C^{\infty}(\mathfrak{U} ; \mathfrak{F})$.

ДокАЗАТЕЛЬСТво. Утверждение 1) леммы 2 очевидно, а утверждение 2) проверяется непосредственно. Укажем лишь, что

$$
M_{u}^{\prime}=\left(\begin{array}{ccc}
\Sigma B_{\sigma} & \Sigma B_{\pi} & \mathbf{0} \\
\Pi B_{\sigma} & \Pi B_{\pi} & -I \\
\mathbf{0} & C & \mathbf{0}
\end{array}\right)
$$

где $B_{\sigma}\left(B_{\pi}\right)$ - частная производная $\Phi$ реше оператора $B$ в точке $u_{\sigma}+u_{\pi}$ по $u_{\sigma}\left(u_{\pi}\right)$.

Положим $f=\left(f_{\sigma}, f_{\pi}, 0\right)$ и, тем самьм, закончим редукцию задачи $(2),(4)$ к задаче $(5),(6)$. Далее проверим условия A1)-A4). Чтобы проверить выполнение A1), обозначим через $A_{\varkappa \sigma}$ сужение оператора $\Sigma A_{\varkappa} \Sigma$ на $\mathbf{H}_{\sigma}^{2}$.

Лемма 3. Пусть выполнены условия леммы 2 , причем $\operatorname{ker} A_{\varkappa \sigma}=\{0\}$. Тогда каждый вектор $\varphi \in \operatorname{ker} L \backslash\{0\}$ имеет точно один $M_{u}^{\prime}$-присоединенный вектор независимо от точки $и \in \mathfrak{U}$. 
ДокАЗАТЕЛЬСтво. Пусть вектор $\varphi \in\left(0,0, \varphi_{p}\right) \in \operatorname{ker} L, \varphi_{p} \notin 0$. Найдем вектор $\psi \in \mathfrak{U}$ такой, что $L \psi=M_{u}^{\prime} \varphi$. Из (17), (19) имеем

$$
A_{\varkappa \sigma} \psi_{\sigma}+\Sigma A_{\varkappa} \psi_{\pi}=0, \quad \Pi A_{\varkappa} \psi_{\sigma}+\Pi A_{\varkappa} \psi_{\pi}=-\varphi_{p}
$$

В силу теоремы Солонникова-Воровича-Юдовича (см. [3]) существует оператор $A_{\varkappa \sigma}^{-1} \in$ $\mathscr{L}\left(\mathbf{H}_{\sigma}, \mathbf{H}_{\sigma}^{2}\right)$. Поэтому из $(20)$ получаем $\psi_{\sigma}=-A_{\varkappa \sigma} \Sigma A_{\varkappa} \psi_{\pi}$. Значит, если $\psi_{\pi}=0$, то $\psi_{\sigma}=0$ и $\varphi_{p}=0$. Итак, $\psi_{\pi} \neq 0$.

Пусть

$$
\widetilde{L}^{-1}=\left(\begin{array}{ccc}
\Sigma A_{\varkappa}^{-1} \Sigma & \Sigma A_{\varkappa}^{-1} \Pi & 0 \\
\Pi A_{\varkappa}^{-1} \Sigma & \Pi A_{\varkappa}^{-1} \Pi & 0 \\
\mathbf{0} & \mathbf{0} & \mathbf{0}
\end{array}\right)
$$

Поскольку

$$
\widetilde{L}^{-1} L=\left(\begin{array}{ccc}
\Sigma & \mathbf{0} & \mathbf{0} \\
\mathbf{0} & \Pi & \mathbf{0} \\
\mathbf{0} & \mathbf{0} & \mathbf{0}
\end{array}\right) \in \mathscr{L}(\mathfrak{U}), \quad L \widetilde{L}^{-1}=\left(\begin{array}{ccc}
\Sigma & \mathbf{0} & \mathbf{0} \\
\mathbf{0} & \Pi & \mathbf{0} \\
\mathbf{0} & \mathbf{0} & \mathbf{0}
\end{array}\right) \in \mathscr{L}(\mathfrak{F})
$$

то $\psi_{\sigma}=-\Sigma A_{\varkappa}^{-1} \varphi_{p}, \psi_{\pi}=-\Pi A_{\varkappa}^{-1} \varphi_{p}$, а компонента $\psi_{p}$ вектора $\psi$ произвольна.

Далее,

$$
M_{u}^{\prime} \psi=\left(\begin{array}{c}
\Sigma\left(B_{\sigma} \psi_{\sigma}+B_{\pi} \psi_{\pi}\right) \\
\Pi\left(B_{\sigma} \psi_{\sigma}+B_{\pi} \psi_{\pi}\right)-\varphi_{p} \\
C \psi_{\pi}
\end{array}\right)
$$

Поскольку $\psi_{\pi} \neq 0$, то $C \psi_{\pi} \neq 0$ [13]. Отсюда $M_{u}^{\prime} \psi \notin \operatorname{im} L$ независимо от $u \in \mathfrak{U}$.

Итак, условие A1) выполняется, причем $p=1$. Приступим к проверке А2). Обозначим через $A_{\varkappa \pi}$ сужение оператора $\Pi A_{\varkappa \pi}^{-1} \Pi$ на $\mathbf{H}_{\pi}$.

Лемма 4. В условиях леммы 3 оператор $A_{\varkappa \pi}: \mathbf{H}_{\pi} \rightarrow \mathbf{H}_{\pi}^{2}-$ топлинейньй изоморфизм.

ДокАЗАТЕЛЬСтво. По построению оператор $A_{\varkappa \pi} \in \mathscr{L}\left(\mathbf{H}_{\pi}, \mathbf{H}_{\pi}^{2}\right)$. Установим его биективность. Пусть $f_{\pi} \in \operatorname{ker} A_{\varkappa \pi}$. Тогда $A_{\varkappa}^{-1} f_{\pi}=u_{\sigma} \in \mathbf{H}_{\sigma}^{2}$, т.е. $f_{\pi}=A_{\varkappa} u_{\sigma}$. Отсюда $A_{\varkappa \sigma} u_{\sigma}=0$; значит, $u_{\sigma}=0$, поэтому $f_{\pi}=0$. Итак, оператор $A_{\varkappa \pi}$ инъективен.

Теперь установим его сюръективность. Пусть $u_{\pi} \in \mathbf{H}_{\pi}^{2}$. Положим $u_{\sigma}=-A_{\varkappa \sigma}^{-1} \Sigma A_{\varkappa} u_{\pi}$ $\in \mathbf{H}_{\sigma}^{2}$. Тогда

$$
\Sigma A_{\varkappa} u_{\sigma}+\Sigma A_{\varkappa} u_{\pi}=0, \quad \Pi A_{\varkappa} u_{\sigma}+\Pi A_{\varkappa} u_{\pi}=f_{\pi}
$$

Отсюда $A_{\varkappa}\left(u_{\sigma}+u_{\pi}\right)=f_{\pi}$, т.е. $u_{\sigma}+u_{\pi}=A^{-1} f_{\pi}, u_{\pi}=A_{\varkappa \pi} f_{\pi}$. Доказательство завершено.

В силу леммы 2 оператор $L$ из (17) бирасшепляющий. Положим $\mathfrak{U}_{0}^{0}=\operatorname{ker} L, \operatorname{coim} L=$ $\mathbf{H}_{\sigma}^{2} \times \mathbf{H}_{\pi}^{2} \times\{0\}$. Построим линеалы

$$
\begin{gathered}
\mathfrak{F}_{0}^{0}=M_{u_{0}}^{\prime}\left[\mathfrak{U}_{0}^{0}\right]=\{0\} \times \mathbf{H}_{p} \times\{0\}=\{0\} \times \mathbf{H}_{\pi} \times\{0\} \subset \operatorname{im} L, \\
\mathfrak{U}_{1}^{0}=\widetilde{L}^{-1}\left[\mathfrak{F}_{0}^{0}\right]=\Sigma A_{\varkappa}^{-1}\left[\mathbf{H}_{p}\right] \times A_{\varkappa \pi}\left[\mathbf{H}_{p}\right] \times\{0\}=\Sigma A_{\varkappa}^{-1} A_{\varkappa \pi}^{-1}\left[\mathbf{H}_{\pi}^{2}\right] \times \mathbf{H}_{\pi}^{2} \times\{0\} .
\end{gathered}
$$

В силу леммы 4

$$
\mathfrak{F}_{1}^{0}=M_{u_{0}}^{\prime}\left[\mathfrak{U}_{1}^{0}\right]=\Sigma B_{0} A_{\varkappa}^{-1}\left[\mathbf{H}_{p}\right] \times \Pi B_{0} A_{\varkappa}^{-1}\left[\mathbf{H}_{p}\right] \times C A_{\varkappa}^{-1}\left[\mathbf{H}_{p}\right]
$$


Пусть $\widetilde{C}$ - сужение оператора $C$ на $\mathbf{H}_{\pi}^{2}$. Поскольку существует оператор $\widetilde{C}^{-1}[13]$, в силу леммы 4

$$
\mathfrak{F}_{1}^{0}=\Sigma B_{0} A_{\varkappa}^{-1} A_{\varkappa \pi}^{-1} \widetilde{C}^{-1}\left[\mathbf{H}_{p}\right] \times \Pi B_{0} A_{\varkappa}^{-1} A_{\varkappa \pi}^{-1} \widetilde{C}^{-1}\left[\mathbf{H}_{p}\right] \times \mathbf{H}_{p}
$$

Здесь и вьше $B_{0}-$ производная $\Phi$ реше оператора $B$ в точке $u_{\sigma}^{0}+u_{\pi}^{0}$, а оператор $\widetilde{L}^{-1}$ определен в (21).

Построим операторы

$$
P_{0}=\left(\begin{array}{ccc}
\mathbf{0} & \mathbf{0} & \mathbf{0} \\
\mathbf{0} & \mathbf{0} & \mathbf{0} \\
\mathbf{0} & \mathbf{0} & \Pi
\end{array}\right), \quad P_{1}=\left(\begin{array}{ccc}
\mathbf{0} & P_{1}^{12} & \mathbf{0} \\
\mathbf{0} & \Pi & \mathbf{0} \\
\mathbf{0} & \mathbf{0} & \mathbf{0}
\end{array}\right)
$$

где $P_{1}^{12}=\Sigma A_{\varkappa}^{-1} A_{\varkappa \pi}^{-1} \Pi$

$$
Q_{0}=\left(\begin{array}{ccc}
\mathbf{0} & \mathbf{0} & \mathbf{0} \\
Q_{0}^{21} & \Pi & Q_{0}^{23} \\
\mathbf{0} & \mathbf{0} & \mathbf{0}
\end{array}\right), \quad Q_{1}=\left(\begin{array}{ccc}
\mathbf{0} & \mathbf{0} & Q_{1}^{13} \\
\mathbf{0} & \mathbf{0} & Q_{1}^{23} \\
\mathbf{0} & \mathbf{0} & \Pi
\end{array}\right),
$$

где $Q_{1}^{13}=\Sigma B_{0} A_{\varkappa}^{-1} A_{\varkappa \pi}^{-1} \widetilde{C}^{-1} \Pi, Q_{1}^{23}=\Pi B_{0} A_{\varkappa}^{-1} A_{\varkappa \pi}^{-1} \widetilde{C}^{-1} \Pi, Q_{0}^{21}=-\Pi A_{\varkappa} A_{\varkappa \sigma}^{-1} \Sigma, Q_{0}^{23}=$ $-Q_{0}^{21} Q_{1}^{13}-Q_{1}^{23}$. Нетрудно заметить, что операторы $P_{k} \in \mathscr{L}(\mathfrak{U})$ и $Q_{k} \in \mathscr{L}(\mathfrak{F}), k=0,1,-$ проекторы, построенные в $(22),(23)$, причем $\operatorname{im} P_{k}=\mathfrak{U}_{k}^{0}, \operatorname{im} Q_{k}=\mathfrak{F}_{k}^{0}, k=0,1$, и $P_{0} P_{1}=P_{1} P_{0}=\mathbf{0}, Q_{0} Q_{1}=Q_{1} Q_{0}=\mathbf{0}$. Поскольку $\operatorname{ker} Q_{1}=\operatorname{im}\left(I-Q_{1}\right)=\operatorname{im} L$, то $\mathfrak{F}_{1}^{0} \oplus \operatorname{im} L=\mathfrak{F}$, т.е. условие A2) выполнено.

Условие А3) вьполняется, поскольку $Q_{1} f=Q_{1}\left(f_{\sigma}, f_{\pi}, 0\right)=(0,0,0)$.

Для проверки условия А4) построим множество $\widetilde{\mathfrak{U}}=\left\{u \in \mathfrak{U}: P_{1} u=\right.$ const $\}=\{u \in \mathfrak{U}$ : $u_{\pi}=$ const $\}$. В нашем случае условие A4) состоит из единственного равенства

$$
Q_{1} M(u)=\left(\begin{array}{c}
Q_{1}^{13} C\left(u_{\sigma}+u_{\pi}\right) \\
Q_{1}^{23} C\left(u_{\sigma}+u_{\pi}\right) \\
C\left(u_{\sigma}+u_{\pi}\right)
\end{array}\right)=\left(\begin{array}{c}
0 \\
0 \\
0
\end{array}\right)
$$

которое вьполняется тождественно [13], если $u_{\pi}=0$. Итак, если положить $\widetilde{\mathfrak{U}}=\{u \in \mathfrak{U}$ : $\left.u_{\pi}=0\right\}$, то А4) вьполняется.

Построим множество $\mathfrak{B}^{t}$. Согласно теореме 1

$$
\mathfrak{B}^{t}=\left\{(u, t) \in \widetilde{\mathfrak{U}} \times \mathbb{R}: Q_{0}(M(u)+f(t))=0\right\} .
$$

Поскольку

$$
\begin{aligned}
Q_{0} & \left(M\left(\begin{array}{c}
u_{\sigma} \\
0 \\
u_{p}
\end{array}\right)+\left(\begin{array}{c}
f_{\sigma}(t) \\
f_{\pi}(t) \\
0
\end{array}\right)\right)=\left(\begin{array}{l}
0 \\
0 \\
0
\end{array}\right) \\
& \Longleftrightarrow\left(Q_{0}^{21} \Sigma+\Pi\right) B\left(u_{\sigma}\right)-u_{p}+Q_{1}^{21} f_{\sigma}(t)+f_{\pi}(t)=0
\end{aligned}
$$

и

$$
Q_{0}^{21} \Sigma+\Pi=A_{\varkappa \pi}^{-1} \Pi A_{\varkappa}^{-1} \Sigma+A_{\varkappa \pi}^{-1} \Pi A_{\varkappa}^{-1} \Pi=A_{\varkappa \pi}^{-1} \Pi A_{\varkappa}^{-1},
$$


имеет место

$$
\mathfrak{B}^{t}=\left\{(u, t) \in \mathfrak{U} \times \mathbb{R}: A_{\varkappa \pi}^{-1} \Pi A_{\varkappa}^{-1}\left(B\left(u_{\sigma}\right)+f_{\sigma}(t)\right)+f_{\pi}(t)=u_{p}, u_{\pi}=0\right\} .
$$

Для доказательства (24) заметим, что

$$
\Pi A_{\varkappa}^{-1} A_{\varkappa \sigma} \Sigma+\Pi A_{\varkappa}^{-1} \Pi A_{\varkappa} \Sigma=\Pi A_{\varkappa}^{-1}\left(\Sigma A_{\varkappa}+\Pi A_{\varkappa}\right) \Sigma=0 .
$$

Отсюда

$$
\begin{gathered}
\Pi A_{\varkappa}^{-1} A_{\varkappa \sigma} \Sigma=-A_{\varkappa \pi} \Pi A_{\varkappa} \Sigma, \quad A_{\varkappa \pi}^{-1} \Pi A_{\varkappa}^{-1} A_{\varkappa \sigma} \Sigma=-\Pi A_{\varkappa} \Sigma, \\
A_{\varkappa \pi}^{-1} \Pi A_{\varkappa}^{-1} \Sigma=-\Pi A_{\varkappa} A_{\varkappa \sigma}^{-1} \Sigma=Q_{0}^{21} \Sigma .
\end{gathered}
$$

Доказана

ТЕОРема 2. Пусть выполнены условия леммы 3 . Пусть $f \in C^{\infty}\left(\mathbb{R} ; L^{2}(\Omega)\right)$, $a\left(\mathbf{v}_{0}, 0\right) \in \mathfrak{B}^{0}(25)$. Тогда для некоторого $t_{0}=t_{0}\left(\mathbf{v}_{0}\right)$ существует единственное решение $(\mathbf{v}, \mathbf{p})$ задачи (2), (4) такое, что $\mathbf{v} \in C^{\infty}\left(\left(-t_{0}, t_{0}\right) ; \mathbf{H}_{\sigma}^{2}\right), \mathbf{v}_{\pi}=0, a$ $\mathbf{p}=A_{\varkappa \pi}^{-1} \Pi A_{\varkappa}^{-1}\left(B\left(\mathbf{v}_{\sigma}\right)+f_{\sigma}(t)\right)+f_{\pi}(t)$.

ЗАмЕчАниЕ 4. Изложенный здесь формализм задачи (2), (4) отличается от формализма, рассмотренного в работах [2], [3], [14].

ЗАмЕЧАнИЕ 5. Если не заниматься построением множества $\mathfrak{B}^{t}$, то теорема 2 может быть доказана проще [6], [7].

\section{СПИСОК ЦИТИРОВАННОЙ ЛИТЕРАТУРЫ}

[1] Осколков А. П. Начально-краевые задачи для уравнения движения жидкостей Кельвина-Фойгта и жидкостей Олдройта // Тр. МИАН. 1988. Т. 179. С. 126-164.

[2] Свиридюк Г.А., Сукачёва Т.Г. Фазовые пространства одного класса операторных уравнений // Дифференц. уравнения. 1990. Т. 26. № 2. С. 250-258.

[3] Свиридюк Г.А., Сукачёва Т. Г. Задача Коши для одного класса полулинейных уравнений типа Соболева // Сиб. матем. ж. 1990. Т. 31. № 5. С. 109-119.

[4] Осколков А. П. К теории жидкостей Фойгта // Записки научн. семин. ЛОМИ. 1980. Т. 96. C. 233-236.

[5] Свиридюк Г.А. Полулинейные уравнения типа Соболева с относительно ограниченными операторами // Докл. АН СССР. 1991. Т. 318. № 4. С. 828-831.

[6] Свиридюк Г. А. Об одной модели динамики несжимаемой вязко-упругой жидкости // Изв. вузов. Матем. 1988. № 1. С. 74-79.

[7] Свиридюк Г.А. О многообразии решений одной задачи несжимаемой вязкоупругой жидкости // Дифференц. уравнения. 1988. Т. 24. №10. С. 1846-1848.

[8] Ландау Л.Д., Лифшиц Е. М. Гидродинамика. 3-е изд. М.: Наука, 1986.

[9] Борисович Ю. Г., Звягин В. Г., Сапронов Ю. И. Нелинейные фредгольмовы отображения и теория Лере-Шаудера // УМН. 1977. Т. 32. № 4. С. 3-54.

[10] Вайнберг М. М., Треногин В.А. Теория ветвления решений нелинейных уравнений. M.: Наука, 1969.

[11] Levine H. A. Some nonexistance and instability theorems for solutions of formally parabolic equation of the form $P u_{t}=-A u+F(u) / /$ Arch. Rational Mech. Anal. 1973. V. 51. № 5 . P. 371-386.

[12] Ленг С. Введение в теорию дифференцируемых многообразий. М.: Мир, 1967.

[13] Капитанский Л. В., Пилецкас К. Н. О некоторых задачах векторного анализа // Записки научн. семин. ЛОМИ. 1984. Т. 138. С. 65-85.

[14] Осколков А. П. Нелокальные проблемы для одного класса нелинейных операторных уравнений, возникающих в теории уравнений типа С.Л. Соболева // Записки научн. семин. ЛОМИ. 1991. Т. 198. С. 31-48. 